\title{
HACIA LA INVESTIGACIÓN MULTIDISCIPLINAR E INTERDISCIPLINAR: REFLEXIÓN SOBRE LA APLICACIÓN DE METODOLOGÍAS PARTICIPATIVAS Y LA INVESTIGACIÓN ACCIÓN PARTICIPATIVA EN LAS CIENCIAS SOCIALES.
}

Norling S. Solís Narváez

\begin{abstract}
Resumen:
La Investigación Acción Participativa (IAP) y las Metodologías Participativas (MP) en las investigaciones sociales ban permitido grandes avances en el desarrollo de las investigaciones. La investigación multidisciplinar e interdisciplinar es un campo que por distintas razones ba sido difícil trabajar de manera armónica. Lo anterior lleva a la reflexión en torno a las metodologías que permitan el trabajo en equipo de investigadores provenientes de disciplinas diversas. En esto radica la importancia del uso de las Metodologías Participativas (MP) y la Investigación Acción Participativa (IAP) como metodología investigativa con enfoque multidisciplinar e interdisciplinar, y como posible solución al problema de relación investigativa entre las disciplinas.

Este trabajo se realizó aplicando técnicas de investigación documental, y análisis de estudios de casos donde se aplicó la investigación multidisciplinar e interdisciplinar. El objetivo central es analizar la importancia de la Metodologías Participativas (MP) y la Investigación Acción Participativa (IAP), encaminado a fortalecer los enfoques de las investigaciones. De ninguna forma se pretende descalificar los procesos de investigación, si no reflexionar para futuras propuesta metodológica entorno a la investigación interdisciplinar y multidisciplinar.
\end{abstract}

Palabras clave: Investigación multidisciplinar, investigación cualitativa, investigación cuantitativa, Investigación Acción Participativa, individualismo y colectivismo

\begin{abstract}
:
Participatory Action Research (PAR) and the Participatory Methodologies (MP) in social research bas enabled great advances in research development. Interdisciplinary and multidisciplinary research bave been difficult to work barmoniously due to several reasons. This leads us to think over metbodologies to be used by multidisciplinary team research, which bighlight importance of participatory methodologies (MP) and participatory action (IAP) to strengthen this research approaches, and also contribute to solve research problems witbin disciplines. This work was conducted through desk research and analysis of case of studies where multidisciplinary and interdisciplinary research was applied. The main objective was to analyze the importance of Participatory Methodologies (MP) and Participatory Action, to strengthen the research approaches. It is not expected to discredit the research process, but think over methodological proposal for interdisciplinary and multidisciplinary research.
\end{abstract}

Keywords: Multidisciplinary research, qualitative research, quantitative research, participatory action research, individualism and collectivism

\section{Introducción}

Las ciencias sociales, han tenido un giro determinante en el campo de las investigaciones. Durante los últimos años se ha dado un incremento en el uso multidisciplinar de las investigaciones. Esencialmente dentro de las investigaciones de desarrollo humano, o bien de problemas multifactorial (como la pobreza) en el cuyas respuesta parten de distintas ópticas científicas.

Dentro de las tendencias investigativa de los organismos, instituciones o asociaciones, se encuentran las investigaciones multidisciplinares. Hacia la investigación Multidisciplinar e interdisciplinar: Reflexión sobre la ..
En este sentido, es una propuesta metódica que no diluya (o margina) los enfoques de cada ciencia o disciplina, al ponerse en marcha una investigación bajo este enfoque, sino que busca responder desde distintas ópticas (espacios de saberes) a una misma pregunta o problema central.

Las disciplinas sociales y humanísticas por su orientación científica están en constante renovación, reformulación, argumentación, o bien buscan la creación de nuevos conocimientos usando sus propias herramientas, técnicas, métodos y teorías. Sin embargo hay un punto en común entre cada una de las disciplinas, no existe una en particular, que

Norling S. Solís Narváez 
responda de forma íntegra y global a un fenómeno. Lo que quiere decir que de manera general existe un vacío, dentro de las disciplinas, pudiendo ser completada por otra disciplina si fuese el caso. En la práctica, el completar ese vacío sería investigación multidisciplinar o interdisciplinar según el caso de consulta entre los investigadores (Kapila \& Moher, 1995).

Para efecto de las investigaciones multidisciplinar e interdisciplinar existe un proceso metódico a tomar en cuenta: la congruencia de las actitudes y aptitudes de los investigadores, aunque en esencia los mismos presenten diferencias bien delimitadas. Para lograr el trabajo en equipo multidisciplinar e interdisciplinar, la propuesta seria tener congruencia de los métodos y técnicas de investigación de cada disciplina, consultas previas.

En una experiencia de trabajo de campo multidisciplinar o interdisciplinar, lo ideal sería que cada disciplina tenga cabida sin protagonizar ni subestimar cada trabajo como investigador. Para lograr un trabajo multidisciplinar, el solo hecho de unir todas las reflexiones en apartados cumple el propósito pero si se quiere llegar más lejos como el caso interdisciplinar, esto demanda relaciones previas y posteriores de los investigadores para analizar un mismo problema entre todos. $Y$ para lograr "lo ideal" de la investigación interdisciplinar y multidisciplinar, es pertinente tener una metodología que logre unificar esfuerzo de los investigadores, una metodología ideal serían las participativas.

\section{El "individualismo" en las investigaciones multidisciplinares e interdisciplinares}

Para las investigaciones multidisciplinares e interdisciplinares hay algunos aspectos esenciales a tomar en cuenta cuando se habla de ir al contexto a realizar trabajo de campo. Desde las academias o escuelas, se enseña a los discentes un método, técnicas y herramientas que deben utilizar para realizar estudios de campo. Desde sus inicios los investigadores conocen y aprenden los métodos y técnicas que su disciplina usa, luego esto se vuelve parte esencial de su identidad como investigador.

Al paso y por la forma cómo fue aprehendido el desarrollo de cada investigador, este presenta un entusiasmo elevado por realizar todos sus trabajos con lo que aprendió al momento de encontrarse con otro investigador y ver diferencias, surgiendo preguntas como, "imi método y metodología de investigación es suficiente?, esto lo puedo hacer solo,

Norling S. Solís Narváez no necesito de otro investigador". Estas frases son supuestos, sin embargo en las praxis son emociones y sentimientos que aflora entre los investigadores por creer que alguien les devaluará su metodología de investigación.

Para lograr una meta multidisciplinar en las investigaciones, se necesita de actitud propositiva en el investigador. Existe un fenómeno dentro del gremio investigador-académico que disminuye la calidad de los trabajos multidisciplinares: el celo profesional. Parece ser un asunto simple y sin fundamento pero analizando los procesos en los que se llevan a cabo las investigaciones multidisciplinares, este defecto actitudinal puede ser un obstáculo para el optimizar el desarrollo de las investigaciones multidisciplinares, sin obviar otro caso particulares que incidan en el progreso de las investigaciones.

Los investigadores generalmente y por naturaleza humana tratan de poner e incluso imponer su sello a cada producto investigativo que realiza. En este sentido, cada autor tiene un sello personal para diferenciarse del resto. Sin embargo dentro de las investigaciones multidisciplinares, el reto es precisamente ese: evitar que el sesgo personal se anteponga a los intereses colectivos, aunque parezca paradójico, sin perder la esencia de cada disciplina, y dando respuesta desde distintas ópticas a un mismo fenómeno en estudio.

Desde las investigaciones tradicional y clásica se ha superado los límites entre el investigador e investigado. Ya el investigador no es el que está arriba ni debajo del investigado si no está a un mismo nivel donde observa y es observado. Ya la posición positivista en este caso ha sido superada en gran manera. (Gil \& Martinez Perez, 2014).

Ahora bien, el punto crítico se encuentra en superar la perspectiva y límites entre los investigadores involucrados en un trabajo multidisciplinar. Lograr en todo caso un punto de equilibrio entre los investigadores al momento de estar en campo y trabajar con un mismo contexto y sujetos de investigación.

\section{Enfoque cualitativo, cuantitativo y mixto en las investigaciones sociales}

Los enfoques de las investigaciones sociales han evolucionado paralelo al mismo desarrollo de las disciplinas y su objeto de estudio. Lo que infiere de cierto modo, que lo que empezó como absolutismo cualitativo y absolutismo cuantitativo (Orti en Hacia la investigación Multidisciplinar e interdisciplinar: Reflexión sobre la ... 
Delgado \& Gutiérrez, 1995), hoy se puede abordar como complementariedad y de forma más acertada como investigación con enfoque mixto.

Los enfoques cualitativos y cuantitativos son percibidos como antagónicos aunque en la práctica ambos mutuamente se complementan en el campo de la investigación. Ambos enfoques tienen orígenes y desarrollos diferentes con sus particularidades, pero a su vez dentro de la nueva tendencia o prácticas de investigación son o incluyente o necesarios para comprender la totalidad de un fenómeno o hecho que en el que se trabaja.

Cada vez más los investigadores pertenecientes a campo de las ciencias sociales o humanísticas se dan cuenta de la necesidad de incluir en sus trabajos los enfoques cualitativos, como la observación, la entrevista, historias de vidas y enfoques cuantitativos, recolectados datos a partir de encuestas y otras técnicas estadísticas o numéricas.

En relación a las fronteras entre investigación cuantitativa y cualitativa y sus conexiones complejas dentro de las ciencias sociales, es interesante lo que plantea Deslauriers, quien se apoya en otros autores (Strauss y Corbin) para fundamentar su tesis:

La investigación cualitativa no se caracteriza por los datos, porque también estos pueden ser cuantificados, sino más bien por su método de análisis que no es matemático (Strauss y Corbin,1980:117-118). La investigación cualitativa es, ante todo, intensiva en lo que ella se interesa: en los casos y en las muestras, si bien limitadas, pero estudiadas en profundidad. (Deslauriers, 2004, pág. 6).

Además la investigación cualitativa por su re flexibilidad y flexibilidad permite "regresar a etapas previas." (Sampieri, Fernandez Collado, \& Baptista Lucio, 2010, pág. 8) En esencia es un enfoque más flexible y permite la profundidad de análisis en los estudios que son llevados a cabo.

Por otro lado plantea Sampiere (2010) establece una estructura a tener en cuenta en la investigación con enfoque cuantitativo:

El enfoque cuantitativo (que representa, como dijimos, un conjunto de procesos) es secuencial y probatorio. Cada etapa precede a la siguiente y no podemos "brincar o eludir" el orden es riguroso, aunque, desde luego, podemos redefinir alguna fase. Parte de una idea, que va acotándose y, una vez delimitada, se derivan objetivos y preguntas de investigación, se revisa la literatura y se construye un marco o una perspectiva teórica. De las preguntas se establecen hipótesis y determinan variables; se desarrolla un plan para probarlas (diseño); se miden las variables en un determinado contexto; se analizan las mediciones obtenidas (con frecuencia utilizando métodos estadísticos), y se establece una serie de conclusiones respecto de la(s) hipótesis.(Sampieri, Fernandez Collado, \& Baptista Lucio, 2010, pág. 4).

Los enfoques anteriormente planteados tienen en si un progreso en cada uno de sus campos de estudios, desde las técnicas que usan, hasta los procesamientos de datos recolectados. Sin embargo existe la última etapa de pautas a la multidisciplinaridad, la cual se identifica con la investigación con enfoques mixto.

Acerca de la investigación relacionada con enfoque mixto, Sampieri (2010) señala que "la meta de la investigación mixta no es reemplazar a la investigación cuantitativa ni a la investigación cualitativa, sino utilizar las fortalezas de ambos tipos de indagación combinándolas y tratando de minimizar sus debilidades potenciales." (Sampieri, Fernandez Collado, \& Baptista Lucio, 2010, pág. 586).

Precisamente en todos los procesos de investigación, lo ideal al momento de combinar enfoques de investigación, (incluso el potencial recurso humano), es potenciar las fortalezas disminuyendo o mitigando las posibles debilidades.

No es reemplazar ni uno ni otro, si no encontrar en ambos un punto de equilibrio que permita un resultado más acertado, objetivo, profundo y óptimo de alguna investigación en concreto.

El planteamiento anterior es válido para las investigaciones multidisciplinares porque el objetivo es lograr entre los investigadores un punto de equilibrio, para potenciar las fortalezas de cada investigador y disminuir sus debilidades. En otras palabras, es encontrar el punto de congruencia para hacer investigación multidisciplinar.

Por ello es importante tener en cuenta que está superado el distanciamiento entre un enfoque y otro (cualitativo y cuantitativo) siendo el enfoque mixto el más utilizado en la actualidad dentro de los procesos de investigación.

Esto sugiere que puede disminuir el distanciamiento entre los investigadores para lograr la anhelada investigación multidisciplinar. 


\section{Enfoque Investigación Acción (IAP) y Metodologías Participativas (MP) en las investigaciones sociales}

Las investigaciones con enfoque participativo aunque no se tiene la certeza de su origen, múltiples investigadores sociales infieren que estas metodologías nace dentro de los movimientos sociales y procesos informales de formación pedagógica (Freire, 1968).

Algunos autores sugieren que las metodologías participativas y específicamente la IAP tiene su "origen y evolución (...) en los años 70, en un clima de auge de las luchas populares y ante el fracaso de los métodos clásicos de investigación en el campo de la intervención social" (Eizagirre \& Zabala, 2006)

Precisamente tiene una connotación de inclusión popular, es decir, una metodología en la que el investigador logra una participación directa con su sujeto de investigación permitiendo más discusiones dentro del proceso investigativo.

Lo que empezó como una alternativa para salir del esquema de la educación formal, no era importante para los procesos de investigación en sus premisas. Se percibía como una metodología que se posicionaría en un rango considerable, pero sin llegar a calar de manera sustantiva o al menos no más importante que el modelo occidental de investigación.

La investigación acción participativa (IAP) es considerada como una forma más específica de las metodologías participativas'. Todo este concepto participativo en las investigaciones tiene,

...sus precedentes se hallan en el concepto de "investigación-acción" acuñado por Kurt Lewin en 1944, entendido como un proceso participativo y democrático llevado a cabo con la propia población local, de recogida de información, análisis, conceptualización, planificación, ejecución y evaluación. Se trataba de una propuesta que rompía con el mito de la investigación estática y defendía que el conocimiento se podía llevar a la esfera de la práctica, que se podían lograr de forma simultánea avances teóricos, concienciación y cambios sociales. (Eizagirre \& Zabala, 2006)

Precisamente el objetivo real y concreto de las metodologías participativas es lograr el empoderamiento de los sujetos de investigación y como lo plantea Geilfus (2002) a "los beneficiarios" - "comunidad". Para comprender las metodologías participativas se debe aclarar su significado lo cual se observa de manera concreta en la praxis.

Para Geilfuls, la participación "es un proceso mediante el cual la gente puede ganar más o menos grados de participación en el proceso de desarrollo." (Geilfus, 2002, pág. 1). Precisamente esa es la idea de las metodologías participativa, los que se encuentran en el contexto o campo de acción tengan participación directa en el proceso ${ }^{2}$ investigativo o de desarrollo.

Para el desarrollo de la metodología participativa se "combina dos procesos, el de conocer y el de actuar. implicando en ambos a la población cuya realidad se aborda." (Eizagirre \& Zabala, 2006). Necesariamente son dos puntos importantes en cualquier proceso de investigación, primero el conocer sobre lo que se investiga, requiere una preparación anticipada, consistente en la estructuración del protocolo de investigación o bien del plan de acción en el campo de investigación.

Y segundo el actuar, que requiere sobre todo un proceso integral entre el investigador e investigado. La importancia que tienen las metodologías participativas es que "proporciona a las comunidades y a las agencias de desarrollo un método para analizar y comprender mejor la realidad de la población (sus problemas, necesidades, capacidades, recursos), y les permite planificar acciones y medidas para transformarla y mejorarla." (Eizagirre \& Zabala, 2006)

Esencialmente la transformación y/o mejoramiento de la comunidad o sujetos de investigación no es dado, ni proporcionado por el ingenio del investigador, aunque si aporta al respecto. Los constructores del cambio son los mismos actores sociales de la comunidad. El investigador es un facilitador (Freire, 1970).

Para Phil Bartle la participación de la comunidad es vista como:

un "rasgo principal el adiestramiento, este adiestramiento excede su propósito tradicional, que es la transmisión de conocimiento a los alumnos. El adiestramiento para gestión también incluye la

VeaseGeilfus, Frans. 2002. Las 80 herramientas para el desarrollo participativo. San José: IICA

2. Para Geilfus se debe cumplir un proceso necesario en todo proyecto de desarrollo, la escalera de participación o integración de la comunidad. Pensado por el autor de la siguiente forma: Pasividad suministro de información, participación por consulta, participación por incentivos, participación funcional, participación interactiva y auto-desarrollo. 
toma de conciencia, la transferencia de formación y el estímulo." (Bartle, 2007)

Lo anterior hace referencia de manera específica al desarrollo de una comunidad, o bien conocer a profundidad un problema o fenómeno en específico. Esto no implica para algunos investigadores académicos interesados, una exclusión para producir cambios en la comunidad y nuevos conocimientos. Todo lo contrario, estas metodologías logra de la misma forma, la participación de la comunidad, y permite el análisis a profundidad sobre algún fenómeno, desarrollando una producción importante y considerable de conocimiento.

Lo innovador de estas metodologías participativas es que integra entre sus fundamentos importantes del proceso investigación, a la "acción". Esto incluye varios elementos destacables en su proceso reflexible que permite puntos clave:

(i) Se construye desde y para la práctica, (ii) pretende mejorar la práctica a través de su trasformación, al mismo tiempo que procura comprenderla, (iii) demanda la participación de los sujetos en la mejora de sus propias prácticas, (iv) exige una actuación grupal por la que los sujetos implicados colaboran coordinadamente en todas las fases del proceso de investigación, (v) implica la realización de análisis crítico de las situaciones y (vi) se configura como una espiral de ciclos de planificación, acción, observación y reflexión.(Herreras, 2015)

Es importante resaltar la importancia que ha tenido la metodología participativa en los procesos educativos, principalmente en la educación descolonizadora del "Sur".

En otras palabras, toda la tendencia de las propuestas pedagógicas deben ser propias, a partir de las experiencias particulares del medio y no importando cultura ni modelos educativos eurocéntrico. (SolanoAlpízar, 2015)

Y sobre la metodología participativa dentro de la educación, existen aportes importantes como los hechos desde Paulo Freire (1970), expresada en su obra "La pedagogía del Oprimido". Es una propuesta muy latinoamericanista, apegada a la realidad, concretamente vinculante y observable en el quehacer cotidiano del docente-investigador.

El nuevo profesional, demanda un modelo descolonizador, popular y latinoamericano, expresado en palabras sencillas, por Freire (1970) que los últimos años ha existido un re pensamiento de la educación en torno a la descolonización. (Navarro, 2010).

La propuesta reivindicadora de Freire (1970) plantea que el investigador dialoga con los investigados, con los datos, reflexiona y aporta, armonizando la investigación en un sentido práctico, llevado hasta el contexto. Lo que no surge desde el campo de investigación o mejor dicho por los informantes, es lo aquello discreto o "irrelevante".

Lo esencial de las metodologías participativas o al menos lo que lo interesa en este artículo, es la inclusión de esta metodología en la investigación. Lo que ha evolucionado a una forma más integral de hacer investigación cualitativa, pero aun también incluir entre el proceso de la investigación acción participativa el enfoque cuantitativo que da como resultado el enfoque mixto de las investigaciones.

Las metodologías participativas cuentan con una variedad de herramientas 0 instrumentos para lograr la participación de los actores sociales en las investigaciones. En este trabajo, no se hará mención de todas ellas pero si se pueden mencionar algunas, quizás familiares para aquellos docentes que se dedican al trabajo de investigación, como los grupos focales, grupo de discusión, talleres, entro otros.

Estas herramientas hacen referencia a técnicas de investigación acción participativa las cuales forman parte de las investigaciones sociales. Las disciplinas como Antropología, Historia, Trabajo Social, Derecho, Ciencias Políticas, Comunicación, entro otras, por su naturaleza (y dependiendo el tipo de trabajo que realizarán) tienen contacto directo con los sujetos de la investigación. Usan la técnica de Investigación Acción Participativa y logran cumplir de manera más precisa los objetivos que se proponen.

La Investigación Acción Participativa (IAP) logra de forma precisa y entre otras cosas:

- Relación directa y armoniosa del investigador con los actores sociales.

- Discusión y reflexión de la información brindada por los actores sociales.

- Consentimiento pleno e informado de los actores sociales.

- Observar y ser observado

- Relación cíclica con los actores sociales, es decir, que una vez que visitas la comunidad y aplicas una metodología participativa, el mismo proceso de la investigación te obliga a volver a la comunidad 
y ser recibido con el mismo entusiasmo.

- Estar en contacto con los informantes para la actualización de los datos.

- Contribuir al desarrollo de la comunidad desde la reflexión de los actores sociales.

- Servir como facilitador para otros procesos integrales y de desarrollo comunitario del contexto antes visitado.

La Investigación Acción Participativa y otras formas de investigación social usando métodos participativos, donde se incluyen a los sujetos de investigación como protagonistas, logran cambios importantes en los contextos donde son ejecutados. Es una propuesta de investigación descolonizadora, propia del modelo investigador latinoamericano, donde se involucra al sujeto investigado en el proceso y donde el investigador no es un ser sin sentimientos. Su profesionalidad le permite realizar trabajos con métodos y técnicas de investigación objetivas. Además incorpora su subjetividad dentro del proceso del trabajo, incidiendo con sus reflexiones a un cambio o desarrollo social integro, apegado a la realidad social investigada.

\section{Metodologías participativas en los enfoques interdisciplinar y multidisciplinar de las investigaciones sociales}

Las investigaciones sociales tienen un problema real y concreto: tratan de dar respuesta a fenómenos y/o problemáticas, desde su disciplina, con métodos y técnicas surgidas desde sus propios dominios o experiencias, siendo esto, en alguna medida, una camisa de fuerza. Lo anterior crea un problema al momento de pensar en proyectos de investigaciones inter y multidisciplinares.

Las disciplinas han estructurado y validado métodos y técnicas propias. La experiencia de la realidad cotidiana demanda abrirse a otras disciplinas. Esto sugiere utilizar métodos o técnicas de otra disciplina con el fin único de dar respuesta más certera a las interrogantes planteadas en la investigación.

Lógicamente cada investigador quiere llevar el proceso investigativo desde su enfoque metodológico, permitido y con conciencia de su uso, dentro de la investigación multidisciplinar. Pero al momento de participar en una investigación desde esta perspectiva, ninguna disciplina deberá resaltar ni protagonizar más que otra. En otras palabras, todas estarán en el mismo orden de prioridad para responder al objeto de estudio.
De lo anterior, se sabe por experiencia que es complejo y no exento de dificultades, su puesta en marcha, si los participantes no se coordinan bajo criterios metodológicos comunes. Lo que quiere decir, que debe de establecerse estos criterios comunes, antes del trabajo de campo, con el fin que el equipo esté física, psíquica y técnicamente preparada.

Para comprender los niveles de desafíos para la integración de investigaciones multidisciplinares e interdisciplinares, es necesario manejar las diferencias conceptuales entre ambas. De acuerdo a la especialista Carrasco (2015):

La investigación interdisciplinaria surge en el mundo globalizado como respuesta al avance del conocimiento que demanda una mayor comprensión de la diversidad antropológica, étnica, lingüística y sociocultural, entre otras. En una palabra, la complejidad de la realidad humana, plantea problemas y busca respuestas con nuevas estrategias que superen la investigación disciplinar. Por ende, la interdisciplinariedad significa una reorientación porque integra varias disciplinas que en una relación simétrica, dinámica e interactiva conjugan perspectivas de análisis propias de cada una para enriquecer la mirada del objeto de estudio. (P.13)

Claramente los desafíos actuales del mundo global demanda investigaciones más integrales que respondan a objetivos concretos y establecidos a partir de un problema o fenómeno de estudio, por ello que las investigaciones interdisciplinares son una herramienta de desarrollo humano. La búsqueda de lograr la simetría de los investigadores en relación a un objeto de estudio es el reto, que debe ser superado, si se pretende lograr avances a nivel de desarrollo humano integral. En teoría estas investigaciones interdisciplinares cumplen criterios de profundidad y acierto más que una investigación mono disciplinar, sin embargo para lograr ese ideal, se debe analizar la interdisciplinaridad desde la práctica.

Y la práctica induce que los términos son confundidos cuando se aborda la investigación en el campo, por ello es importante aclarar que las investigaciones multidisciplinares tienen desarrollo, que la investigación interdisciplinar, porque la segunda demanda del investigador, mayor compromiso y dialogo con el otro investigador, que la multidisciplinaria.

Para ello es importante aclarar porque se cumple más la multidisciplinaridad que interdisciplinaridad.

Hacia la investigación Multidisciplinar e interdisciplinar: Reflexión sobre la ... 
Esto es porque, La realizaci6n de una investigación con varias disciplinas diferentes puede requerir un enfoque multidisciplinario o interdisciplinario. El primero se basa en aportes paralelos de distintas disciplinas, sin que existan necesariamente instancias de consulta entre ellos. El segundo implica una interacci6n de diferentes disciplinas en relaci6n con el problema que se investiga, a través de todo el proceso de investigaci6n, a partir -en el caso idealde la definici6n del problema.(Kapila \& Moher, 1995)

Las investigaciones multidisciplinares como mencionan los autores no implican una relación de dialogo entre cada disciplina. Aunque eso en la práctica resulta siempre un distanciamiento de las mismas disciplinas, lo que sería una investigación escueta porque implica dejar vacíos de congruencia entre mismas. Ciertamente la interdisciplinaridad responde metódicamente al dialogo entre las disciplinas, esto da pautas de reflexión integral, aunque ya se mencionaba anteriormente que en teoría lo ideal es lograr el dialogo, aunque los intereses individuales se anteponen en la práctica. Pero en ello recae la reflexión de este ensayo, lograr discutir entorno al uso adecuado de la investigación interdisciplinar y multidisciplinar.

80 La multidisciplinaria que refiere a la investigación con varias disciplinas no implica trabajos aislados de cada una de ellas. Al contrario deben tener un acercamiento de preparación de instrumentos hasta el procesamiento de los datos recolectados. Pero bien, sin discutir tanto la metodología de esta investigación, también es importante la interpretación interdisciplinar que refiere a mi criterio un poco más de lo planteado por los autores, esta, refiere un dialogo entre las disciplinas pero además un informe con cada reflexión interdisciplinar y métodos casi similares.

Prácticamente las investigaciones multidisciplinares e interdisciplinares "cobra (n) sentido en la medida en que flexibiliza y amplía los marcos de referencia de la realidad, a partir de la permeabilidad entre las verdades de cada uno de los saberes." (Escobar, 2010)Lo que implica claramente un acercamiento real y concreto de las disciplinas para complementar los vacíos inherentes de cada una de ellas.

Como se planteó al comienzo no es diluir la esencia de cada disciplina si no, encontrar un punto medio, de congruencia en el que cada una de las disciplinas que participan de la investigación logren brindar datos importantes para investigación y aún más importante, que exista otra disciplina que brinde los datos importantes que desde la primera no se logró, eso permite la complementariedad, es decir, llenar los vacíos mutuos.

Para lograr la complementariedad entre las disciplinas en una investigación interdisciplinar, debe existir una metodologías que logre la participación de cada disciplina y que permita que cada una aplique sus instrumentos o bien participen con un mismo método pero con criterios particulares de recolección de información.

Para efecto de ello, se plantea que una forma de lograr ese objetivo es haciendo uso de las metodologías participativas. En ella el investigador pasa a un segundo plano, y el protagonista (sujeto de investigación) participa de una metodología donde puede estar un colectivo de investigadores con objetivo común de investigación y estos podrán analizar el mismo fenómeno, en el mismo contexto, con los mismos sujetos pero con criterios distintos de selección y recolección de información.

Se podrán apoyar mutuamente, para que mientras uno está aplicando algún instrumento, el otro toma apuntes si nota alguna reacción interesante en los sujetos de investigación. El caso interesante es que ninguno de los investigadores es más protagonista que otro. Y ambos logran recolectar información de forma satisfactoria.

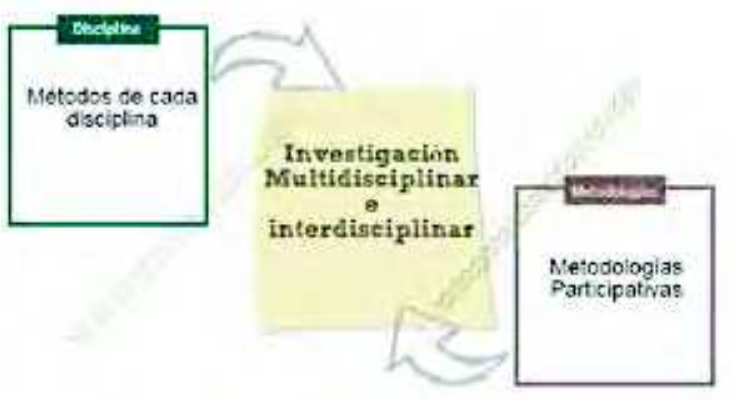

Incluso una vez terminada la sesión metodológica, en el laboratorio se pueden llegar a reflexiones interesantes que aporten a cada disciplina para el análisis del fenómeno.

Cada investigador desde su disciplina podrá recolectar información en el mismo círculo de investigación participativa. Por ejemplo en grupo focal que según Mella (2000) el grupo focal son: "antes que nada una buena técnica para obtener datos de investigación. Al igual que en cualquier proyecto de investigación, con grupos focales uno recolecta y analiza información de manera de poder responder una interrogante de investigación." (Mella, 2000) 
Ejemplo del desarrollo de investigaciones multidisciplinar e interdisciplinar son los proyectos binacionales de la Facultad de Humanidades y Ciencias Jurídicas de la UNAN-Managua. La investigación realizada en Cárdenas, Rivas y Guanacaste Costa Rica tuvo un proceso de preparación para lograr objetivos concretos en la investigación multi e interdisciplinar. Participando de distintas técnicas y métodos de investigación las distintas disciplinas de la facultad participaron en aplicación de instrumentos de distintos enfoques logrando recopilar información en un mismo campo de investigación y sujetos de investigación. De forma que cada investigador podre tener respuesta para el análisis desde su disciplina pero además podrá escuchar lo que responden las personas a las demás preguntas y eso servirle para discutir sus reflexiones.

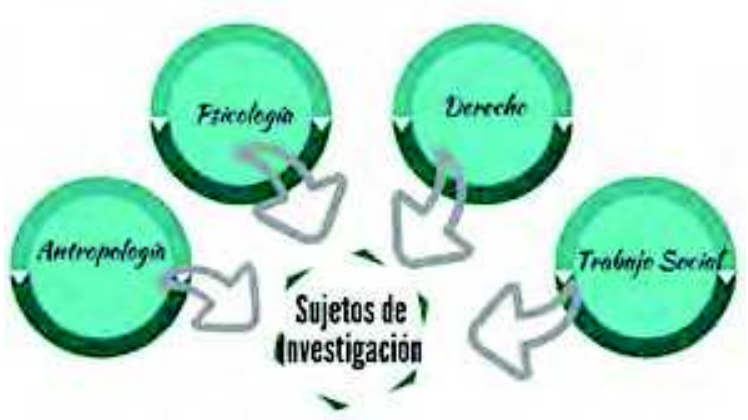

Cuando se analiza este punto de vista de cada reflexión del investigador, lo que se percibe es la relación pareja de los investigadores durante el proceso y desarrollo de la investigación. Durante la aplicación de la técnica participativa, todos están en un mismo nivel de relación con el sujeto de investigación. Y esa simple relación permite la reflexión armoniosa e importante de los investigadores al momento de procesar la información.

Pero para llegar a este punto de equilibrio se necesita tres momentos importantes:

1. Previo a la investigación: a) se plantean las lecturas teóricas, b) selección de muestra, c) selección de técnicas e instrumentos de investigación que deben ser dialogados entre los investigadores, d) creación de instrumentos, e) creación de protocolo de investigación.

2. Durante el trabajo de campo: a) aplicar instrumentos de forma coordinada, b) se debe quedar claro quien aplica primero el instrumento, los demás toman notas de libretas, c) no se debe transmitir a los sujetos de investigación falta de coordinación en el equipo, d) después de terminar la aplicación de instrumentos, en el mismo lugar

Norling S. Solís Narváez u otro, se realiza memoria o diario de campo del día de trabajo, para realizar reflexiones en torno a la aplicación de instrumentos o la pertinencia de los mismos.

3. Posterior a la investigación: a) se realiza la discusión y reflexión de los resultados de forma individual primeramente y posterior en colectivo.

Claramente con esto lo que se quiere lograr es la reflexión en torno a lo planteado, para que se mejoren las propuestas de investigación interdisciplinar y multidisciplinar, este documento debe permitir la reflexión sobre cómo se está realizando las investigaciones con este enfoque y la pertinencia de las metodologías usadas.

\section{Conclusión}

Las investigaciones han logrado un desarrollo importante desde cada enfoque (cualitativo, cuantitativo y mixto) lo que ha permitido la reflexión en torno a la pertinencia del conjunto de los enfoques. De esta manera, ya no se abordan las investigaciones a partir de concepciones o enfoques mutuamente excluyentes, sino todo lo contrario, las investigaciones con enfoque mixtos son la que más se utilizan.

Es una demanda de todas las disciplinas del saber las cuales se ven impelidas por la complejidad y la necesidad de un mundo cada vez más globalizado, que busca respuestas reales y concretas de los diversos fenómenos que aquejan a la humanidad.

Esta nueva perspectiva, abre otra brecha en la investigación social, guiando los esfuerzos de los investigadores a la participación multidisciplinar e interdisciplinar. Claramente han existido fracasos al momento de realizar este tipo de investigaciones, esto responde a distintas causas, desde actitudes egocéntricos de los investigadores, hasta la pertinencia metódica para realizar este tipo de investigación multidisciplinar e interdisciplinar.

Para ello es importante retomar el desarrollo de la investigación acción participativa (IAP) y todas las metodologías participativas (MP) que permite una relación directa con los sujetos de investigación.

En este sentido, la propuesta es lograr la congruencia y punto de equilibrio entre los investigadores, haciendo uso de las metodologías participativas. Esto sin duda es una propuesta que se ha trabajado ya en varias investigaciones con enfoque de desarrollo, y Hacia la investigación Multidisciplinar e interdisciplinar: Reflexión sobre la ... 
que sin duda deben ser analizadas y sistematizadas para lograr la reproducción de esa experiencia donde se logró la investigación interdisciplinar y multidisciplinar haciendo uso de metodologías participativas.

\section{Referencias bibliográficas}

Bartle, P. (08 de Agosto de 2007). Esquema de la estrategia del Programa de Gestion Comunitario. Obtenido de Colectivo de Potenciacion Comunitario : http://cec.vcn. bc.ca/mpfc/modules/stratsks.htm

Carrasco, R. (6 de Agosto de 2015). La investigación interdisciplinaria e internacional. Obtenido de www.unife.edu.pe/centro-investigacion/ boletin/enlaces/1.

Colmenares, A. M., \& Piñero, M. L. (2006). La investigación acción: Una herramienta metodológica heurística para la comprensión y transformación de realidades y prácticas socio-educativas. Laures-Redalyc, 96-114.

Deslauriers, J. (2004). Investigación Cualitativa. Pereira: Editorial Papiro.

Eizagirre, M., \& Zabala, N. (7 de Agosto de 2006). Diccionario de Acción Humanitaria. Obtenido de http://www.dicc.hegoa.ehu. es/listar/mostrar/132

Escobar, Y. C. (2010). Interdisciplinariedad: desafío para la educación superior y la investigación. Revista Luna Azul, 156-169.

Freire, P. (1970). Pedagogía del Oprimido. Argentina: Siglo XXI.

Geilfus, F. (2002). Las 80 Herramientas para el desarrollo participativo. San Jose : IICA.
Gil, J., \& Martinez Perez , S. (2014). La importancia de las relaciones investigador-investigado: el caso de las narrativas de vida profesional. Tendencias Pedagogicas, 225-240.

Herreras, E. B. (10 de Agosto de 2015). Docencia a traves de la investigación-acción. Obtenido de Revista Iberoamericana de Educación: http://www.rieoei.org/ deloslectores/682Bausela.PDF

Kapila, S., \& Moher, R. (1995). Disciplinas interactivas: Principios para la Investigación Interdisciplinaria. Otawa: INTERNATIONAL DEVELOPMENT RESEARCH CENTRE POLICY AND PLANNING GROUP .

Mella, O. (2000). Grupos Focales. Chile : Documento de Trabajo.

Navarro, M. (2010). Estrategias para una educación superior descolonizadora intrae intercultural. Bolivia: FUNPROEIB Andes .

Orti, A. (1995). La confrontacion de modelos y niveles epistemologicos en la genesis e historia de la investigacion social. En J. M. Delgado, \& J. Gutierrez , Métodos y técnicas cualitativas de investigación de investigación en las ciencias sociales (págs. 84-94). Madrid: Sintesis Psicologica .

Sampieri, R. H., Fernandez Collado, C., \& Baptista Lucio, M. (2010). Metodología de la investigación. Quinta edición. México: Interamericana Editores.

Solano-Alpízar, J. (2015). Descolonizar la educación o el desafío de recorrer un camino diferente. Revista Electrónica Educare, 117-129. 